\title{
PCR-based procedures to isolate insertion sites of DNA elements
}

\author{
Bin Yin and David A. Largaespada \\ University of Minnesota, Minneapolis, MN, USA
}

BioTechniques 43:79-84 (July 2007)

doi 10.2144/000112474

\begin{abstract}
During the past several years, retroviral insertional mutagenesis has been fruitfully applied to search for genes/pathways involved in tumorigenesis. Techniques used to identify proviral insertion sites are critical for fulfilling these projects. Although a variety of approaches have been described, an improvement over existing methods is required to recover every possible insertion site for cancer gene discovery, so-called saturation analysis. Here, we have described the development of two ligation-mediated $P C R$ variants, SplinkTA-PCR (STA-PCR) and SplinkBlunt-PCR, for efficient isolation of insertion sites in retrovirus-induced leukemia. Our results demonstrated that these two protocols are complementary to each other and that they are better employed in combination for maximal cloning efficiency. These protocols are easy-to-use, reliable and efficient, and are readily applicable to large-scale cloning of insertion sites of provirus and other integrated DNA elements, as well as for detection and cloning of differential insertions unique to drug-resistant cells.
\end{abstract}

\section{INTRODUCTION}

The recent completion of human and mouse whole genome sequencing projects has revolutionized the way we try to understand biology and will certainly have significant influence on our insights into human diseases at a systemic level. Although the availability of the genomic information provides a great framework for biologists to address a broad range of scientific questions, most of mammalian genes have not been extensively studied yet. In fact, $30 \%-50 \%$ of genes fall into the uncharacterized category (1). Forward genetics represents a classical approach to uncover gene function. One powerful forward genetics strategy is insertional mutagenesis using DNA mutagens, such as retroviruses and transposable elements, which integrate into host genome DNA and can up-regulate or disrupt gene function. Through the identification of the insertion sites of these mutagens, a gene can be assigned a certain phenotype-related function. Recently, insertional mutagenesis has been demonstrated to be a fruitful strategy in genome-wide analysis of cancer genes (2-8). The BXH-2 strain of mice develops acute myeloid leukemia (AML) at a high incidence, providing a good model to identify leukemia genes. The BXH-2 AML arises from infection by a murine leukemia virus (MuLV), which cannot only act as an insertional DNA mutagen to cause leukemia, but also serves as a tag for leukemia-associated genes (9).

Mapping proviral insertion sites (PISs) is important not only for an appreciation of the molecular mechanism of integration, but also for characterization of cellular gene functions using insertion mutagenesis strategy. Several methods have been developed for this purpose, including genomic DNA library screening (10), ligation-mediated PCR (LM-PCR) (3), inverse PCR (11), viral insertion site amplification (VISA) technique (12), and single nucleotide polymorphism (SNP)-based mapping $(13,6)$. Although these methods have been useful and generated large amounts of PISs, their inherent limitations posed either by uneven restriction site distribution, low cloning efficiency, excessive laborious work, or nonspecific amplification need to be overcome in order to facilitate functional genomic studies. Here, we described a PCR-based method allowing for less laborious, less biased, and more efficient mapping of PISs. We applied the method to a large-scale identification of PISs associated with BXH-2 AML. We also assessed the PIS pattern in leukemia cells undergoing drug selection.

\section{MATERIALS AND METHODS}

\section{Cell Lines and Culture}

The BXH-2 AML cell lines used in this study were generated and maintained routinely as previously reported $(14,15)$. Highly Ara-Cresistant cell line $\mathrm{B} 117 \mathrm{H}$ was derived by repetitive exposure of the parental cell line B117 to increasing doses of Ara-C (Pfizer, Kalamazoo, MI, USA) (16). The passage control cell line B117P was simply grown and passaged in regular ASM medium in parallel with selection of drug-resistant cells. The Ara-C sensitivity of control cells and resistant cells was verified by a cytotoxicity assay (16).

\section{Southern Blot Analysis}

Genomic DNA was prepared and analyzed by Southern blot analysis as described before (16). Briefly, $10 \mu \mathrm{g}$ genomic DNA were digested with the restriction enzymes $P v u \mathrm{II}$ and resolved on a $0.9 \%$ agarose gel. The gel was then subjected to depurination, denaturation, neutralization, and blotting. The 400-bp B-ecotropic MuLV-specific probe, $\mathrm{pEnv}$, was generated by cutting the plasmid pAKV5 with restriction enzyme XmaI (17) and labeled with $\alpha-32 \mathrm{P}-\mathrm{dCTP}$ using the random priming labeling approach. The blots were blocked with prehybridization solution for $4 \mathrm{~h}$ at $65^{\circ} \mathrm{C}$ and hybridized with the labeled probes in hybridization solution at $65^{\circ} \mathrm{C}$ overnight. After nonspecific binding was removed with the third washing solution $[0.25 \times$ single-strand conformation polymorphism (SSCP), $0.1 \%$ sodium dodecyl sulfate (SDS)], the blots were exposed to X-ray films (Eastman Kodak, Rochester, NY, USA) at $-70^{\circ} \mathrm{C}$ with intensifying screens.

\section{SplinkTA-PCR Protocol}

For SplinkTA-PCR (STA-PCR), genomic DNA was digested with $7.5 \mathrm{U}$ TaqI per microgram DNA at $65^{\circ} \mathrm{C}$ for $4 \mathrm{~h}$ followed by another 4-h digestion with $7.5 \mathrm{U} B s p L U 11 \mathrm{I}$ and $10 \mathrm{U} B c l \mathrm{I}$ (Roche Diagnostics, Indianapolis, IN, USA) per microgram DNA at $48^{\circ} \mathrm{C}$. In some experiments, different restriction enzyme cocktails were used 
(see Results and Discussion section). The digestion products were purified with QIAquick ${ }^{\circledR}$ PCR Purification kit (Qiagen, Valencia, CA, USA) according to the manufacturer. Briefly, $250 \mu \mathrm{L}$ Buffer PB (Qiagen) were added to each sample, and the mixture was applied to the minicolumns, centrifuged at $11,000 \times g$ for $30 \mathrm{~s}$, washed with $750 \mu \mathrm{L}$ Buffer PE (Qiagen) at $11,000 \times g$ for $30 \mathrm{~s}$, and centrifuged for an additional $60 \mathrm{~s}$ at maximum speed. The DNA was eluted with $\mathrm{H}_{2} \mathrm{O}$. For adenosine addition reactions, each purified, digested DNA sample was incubated with $1 \times$ final concentration of PCR buffer, $1.5 \mathrm{mM} \mathrm{MgCl} 2,0.2$ $\mathrm{mM}$ dNTPs, and $2.5 \mathrm{U}$ Taq DNA polymerase (BIOLASE Technology, Irvine, CA, USA) at $72^{\circ} \mathrm{C}$ for $20 \mathrm{~min}$, followed by another purification using the QIAquick kit. SplinkTA was made by mixing equal molar amounts of oligonucleotides splinkerette (5'-CAT GGTTGTTAGGACTGGAGGGGAA ATCAATCCCCT-3'; the hairpin oligonucleotide) and PrimerLongTA (5'-CC TCCACTACGACTCACTGAAGGGC AAGCAGTCCTAACAACCATGT-3'; the oligonucleotide with an extra T) and incubating for $5 \mathrm{~min}$ at $80^{\circ} \mathrm{C}$ before allowing it to slowly cool down to room temperature $\left(20^{\circ}-25^{\circ} \mathrm{C}\right)$. Then an appropriate amount of SplinkTA was ligated to the extension products using T4 DNA ligase (Promega, Madison, WI, USA) by incubation at $16^{\circ} \mathrm{C}$ for 16-20 h. Using $1 \mu \mathrm{L}$ ligation products as templates, primary PCR was done by adding $1 \times$ final concentration of PCR buffer, $1.5 \mathrm{mM} \mathrm{MgCl}_{2}, 0.2 \mathrm{mM}$ dNTPs, 20 pmol primer AKVp7711 (specific to BXH-2 proviral Env gene), $20 \mathrm{pmol}$ primer P-short (complementary to PrimerLongTA), and 2.5 U Taq DNA polymerase (BIOLASE Technology). PCRs were performed at $95^{\circ} \mathrm{C}$ for 1 min $30 \mathrm{~s}$, followed by 10 cycles of $95^{\circ} \mathrm{C}$ for $5 \mathrm{~s} ; 70^{\circ} \mathrm{C}$ for $3 \mathrm{~min} 10 \mathrm{~s}$ with decreasing $0.5^{\circ} \mathrm{C}$ each cycle; 20 more cycles of $95^{\circ} \mathrm{C}$ for $5 \mathrm{~s} ; 65^{\circ} \mathrm{C}$ for $3 \mathrm{~min}$ $10 \mathrm{~s}$; and final extension at $70^{\circ} \mathrm{C}$ for 10 min. In secondary PCR, all components in each reaction are the same as those in primary PCR, except for using primary PCR products as templates and replacing the pair of primers with nested primers AKVp8712 [specific to $\mathrm{BXH}-2$ proviral long terminal repeat
(LTR)] and Pnest (complementary to PrimerLongTA). The amplification parameters were changed to denature at $95^{\circ} \mathrm{C}$ for $1 \mathrm{~min}$, followed by 11 cycles of $94^{\circ} \mathrm{C}$ for $15 \mathrm{~s} ; 70^{\circ} \mathrm{C}$ for $2 \min 40 \mathrm{~s}$ with decreasing $0.6^{\circ} \mathrm{C}$ each cycle; 25 more cycles of $94^{\circ} \mathrm{C}$ for $15 \mathrm{~s} ; 64^{\circ} \mathrm{C}$ for $30 \mathrm{~s} ; 70^{\circ} \mathrm{C}$ for $2 \mathrm{~min} 10 \mathrm{~s}$; and final extension at $70^{\circ} \mathrm{C}$ for $10 \mathrm{~min}$. After electrophoresis on a $1.5 \%$ agarose gel, individual secondary PCR bands were taken and subsequently cloned into TOPO-TA ${ }^{\circledR}$ vector with TOPO TA Cloning kit (Invitrogen, Carlsbad, CA, USA) for sequencing. Additional primer sequences are available upon request.

\section{SplinkBlunt-PCR Protocol}

Genomic DNA digestion and purification were performed as described in the section entitled SplinkTA-PCR Protocol, using a QIAquick PCR Purification kit. Primer extension was performed by incubating DNA digests in $1 \times$ final concentration of $P f u$ PCR buffer, $0.2 \mathrm{mM}$ dNTPs, 20 pmol biotinylated primer Bio-AKVp7711 (specific to $\mathrm{BXH}-2$ proviral Env gene), and 2.5 U Pfu DNA polymerase (Stratagene, La Jolla, CA, USA) at $95^{\circ} \mathrm{C}$ for $5 \mathrm{~min}, 64^{\circ} \mathrm{C}$ for $30 \mathrm{~min}$, and $72^{\circ} \mathrm{C}$ for $20 \mathrm{~min}$. Then primer extension reaction was purified with a Microcon ${ }^{\circledR}$ YM-30 column (Millipore, Billerica, MA, USA) according to the manufacturer. Briefly, $450 \mu \mathrm{L} \mathrm{H} \mathrm{H}_{2} \mathrm{O}$ were added to each sample, transferred to the column, and centrifuged at $14,000 \times$ $g$ for $10 \mathrm{~min}$. The column was placed upside down and centrifuged for $3 \mathrm{~min}$. To capture the Bio-AKVp7711-derived extension products, $10 \mu \mathrm{L}$ streptavidincoupled magnetic particles (Roche) for each sample were washed with 50 $\mu \mathrm{L} 2 \times$ blocking and washing (BW) buffer (10 mM Tris-HCl, pH 7.5, $1 \mathrm{mM}$ EDTA, $2 \mathrm{M} \mathrm{NaCl}$ ) and resuspended in $40 \mu \mathrm{L} 2 \times \mathrm{BW}$ buffer, mixed with the recovered primer extension products from the Microcon YM-30 column, and incubated at room temperature $\left(20^{\circ}-25^{\circ} \mathrm{C}\right)$ for $3 \mathrm{~h}$. The biotin-bearing DNA fragments were captured using magnetic particle concentrator (MPC) followed by washing twice with $\mathrm{H}_{2} \mathrm{O}$. The captured DNA was ligated to the appropriate amount of SplinkBlunt, which was made by mixing splinkerette with PrimerLong $\left(5^{\prime}\right.$-CCTCCACTACG ACTCACTGAAGGGCAAGCAGTC CTAACAACCATG-3') using the same procedure as in the STA-PCR protocol, with 200 U T4 DNA ligase (Promega). After ligation, the biotin-bearing DNA fragments were captured again in MPC and washed with $100 \mu \mathrm{L} \mathrm{H}_{2} \mathrm{O}$. The recaptured DNA was denatured in $5 \mu \mathrm{L}$ $0.1 \mathrm{~N} \mathrm{NaOH}$ at room temperature for 10 min. Magnetic particles were separated from released DNA fragments with MPC. The supernatant was collected and used as templates for subsequent primary and secondary PCR, which were performed as described in the section entitled SplinkTA-PCR Protocol. Additional primer sequences are available upon request.

\section{Sequence Analysis}

DNA sequence analysis was performed on a basis of Ensembl Mouse Genome database (www.ensembl. org/Mus_musculus). Cellular genomic DNA sequences flanking proviral insertions were mapped by searching against mouse genome database using the nucleotide-nucleotide basic local alignment search tool (BLASTn) algorithm (18).

\section{RESULTS AND DISCUSSION}

We first developed the STA-PCR protocol based on splinkerette-PCR (Figure 1A). Digestion with a mixture of restriction enzymes ( $T a q \mathrm{I}, B c l \mathrm{I}$, and $B s p L U 11 \mathrm{I})$ gave rise to shorter DNA fragments on average than digestion with single enzymes, rendering the fragments more readily amplified by the subsequent PCR (data not shown). To achieve efficient ligation of DNA fragments to splinkerettes, DNA fragments were further modified by Taq DNA polymerases to form sticky ends through the terminal nontemplated adenosine addition reaction $(19,20)$. To test the effect of this modification, the Taq-treated digests, along with untreated digests as a control, were ligated to the pCR2.1TOPO vector (Invitrogen), which bears a thymidine at the end, followed by transformation into competent cells. 
The Taq-treated digests gave rise to a $>4$-fold higher number of kanamycinresistant colonies than did the control digests (data not shown). This result encouraged us to continue to test the whole procedure on $\mathrm{BXH}-2$ leukemia samples. As can be seen in Figure 2, two bands of $190 \mathrm{bp}$ (dotted) and 630 bp appeared in all samples, including the BXH-2 normal tissue control. DNA sequencing indicated that these bands represented two characterized BXH-2 germline-acquired PISs $(14,21)$. The two common bands were also seen consistently in every sample profiled in a large-scale PIS cloning experiment carried out later. In addition, several bands with different sizes can be seen in different samples except the negative controls, which are derived from somatically acquired, tumor-specific PISs.

It should be mentioned that after we submitted our PIS cloning manuscript, the similarity of our idea to add an A with the T-linker design was brought to our attention by one of the reviewers (22). When closely compared with T-linker PCR, our method features the combined advantages of splinkerette adoption, enzyme cocktail use, and fewer experimental steps [adding poly(dT)n to the $3^{\prime}$ end of DNA by $\mathrm{T}(\mathrm{dT})$ and single cycle of PCR with single primer S1 are not required]. Furthermore, since complete digestion with $5^{\prime}$ overhang enzymes is employed, there is in our protocol no limitation arising from the need to perform the incomplete digestion-which can often go beyond control-with $3^{\prime}$ overhang enzymes required by T-linker PCR (22).

We also developed the SplinkBluntPCR protocol by modifying LM-PCR (23) and tested it on the same samples as above. Following DNA digestion with the restriction enzyme cocktail, a biotinylated primer was annealed to the digests to drive primer extension reaction. $P f u$ DNA polymerase was used here to produce blunt-end double-stranded DNA fragments. The extension products were purified with streptavidin magnetic particles prior to ligation to SplinkBlunt. A similar procedure to that in STA-PCR was then performed. As shown in Figure 2, two common bands with the size of 190 and $630 \mathrm{bp}$, and a few extra bands with different sizes can be seen here again. Unexpectedly, one 1.15-kb band was lost from tumor B112 compared with STA-PCR; however, SplinkBlunt-PCR protocol reproducibly obtained an extra 390-bp band from tumor 112. The PCR bands were recovered and sequenced to confirm their specificity.

Comparable PIS cloning efficiencies were observed for STAPCR and SplinkBlunt-PCR when applied to a large-scale PIS cloning on BXH-2 leukemia, but we noticed some differences between them. There were fewer steps involved in the STA-PCR procedure, whereas SplinkBlunt-PCR produced a lower background on a PCR gel enabling PCR band recovery. Because they gave rise to different PIS patterns in some samples, use of both STA-PCR and SplinkBlunt-PCR would improve PIS recovery rate. Although not run in parallel on a large scale, in our experience non-overlapping PISs accounted for approximately $10 \%$ of total PISs recovered. Therefore, for some cases where a full or nearly full recovery is desired, combination of the two protocols is highly recommended. Maximization of their ability to clone PISs could be reached by digesting genomic DNA with more than one combination of restriction enzymes. We have successfully tested various combinations of restriction enzymes, including: (i) AatII, MfeI, NdeI; (ii) HindIII, PvuII, XhoI; or (iii) AseI, $B g$ II, EagI, with different resultant PIS patterns. Furthermore, digestion of ligation products with SacII prior to the primary PCR could help eliminate the interference from the top germline-derived PCR band (600 bp) and increase recovery efficiency (data not shown).

To determine the specificity of these PCR protocols, bands of various sizes were recovered and cloned into TOPO2.1 vector for sequencing. All of the PCR products contained a 141-bp DNA sequence at one end which was identical to the $3^{\prime}$ LTR sequence of the BXH-2 MuLV ecotropic retrovirus, indicating that all of the recovered PCR bands came from junction sites linking retroviral genome to cellular genome. The cellular genomic sequences were different among bands and found to be located at different chromosomal positions (data not shown). In a largescale cloning of PISs using these protocols, the two germline bands seen in Figure 2 appeared again in each sample, proving a consistent amplification of PISs (Figure 3). Moreover, individual tumor DNA samples exhibited various numbers and sizes of bands, indicating that these independent PISs are somatically acquired and are possibly associated with leukemogenesis. Sequencing confirmed the specificity of the amplified PIS products and unraveled their different chromosomal locations. Most PISs hit known genes, some of which have been implicated in leukemia, such as Hoxa 7 and Meisl, whereas the rest have not been described previously.

To further test the power and consistency of these PCR protocols, we profiled PIS pattern in Ara-C-sensitive (B117P) and resistant (B117H) BXH2 leukemia cell lines. A similar PIS pattern was amplified in B117P (10 PCR bands) and in B117H (9 PCR bands). Interestingly, B117P displayed one extra 1.3-kb band (indicated as an arrowhead) compared with $\mathrm{B} 117 \mathrm{H}$ (Figure 4A). These PCR bands were also sequenced to verify their specificity. The same number of insertions was verified by Southern blot analysis using a BXH-2 MuLV-specific probe (Figure 4B). The same number of bands was visualized using each method, indicating a high recovery rate using our PIS cloning protocol.

The proviral insertion recovery rate achieved by the method reported here is a very important part of this discussion. For many of the tumors, proviral insertions were analyzed independently by another group using Southern blot analysis, which showed that 68 somatic proviral insertions were detected in 29 NfI-deficient tumors, giving rise to 2.34 insertions per tumor on average (the calculation was made based on the data available from Table 1 in Reference 10). Strikingly, our protocols generated 129 PISs from 55 Nfl-loss tumors which were equivalent averages to 2.34 PISs per tumor. Although it was possible that some insertions might escape detection by both Southern blot analysis and PCR approach, this result indicates that our technique leads to at least a close- 


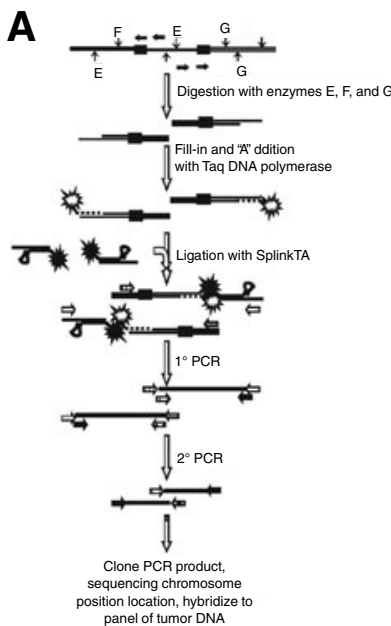

B

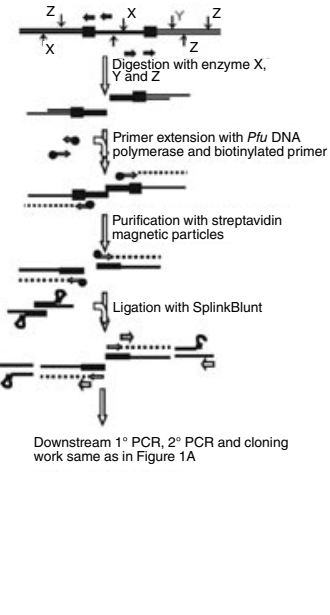

Figure 1. Diagram of strategies for cloning genomic DNA sequence flanking a proviral insertion. (A) SplinkTA-PCR (STA-PCR) protocol. Genomic DNA (horizontal lines) is digested with a cocktail of restriction enzymes (noted as E, F, and G), which have no recognition site within proviral genome sequence (black boxes) downstream to the primers (horizontal arrows) used for primary and secondary PCR. The digestion produces $5^{\prime}$ overhang ends which are subsequently filled in and added an adenosine (open star) at the ends with Taq DNA polymerase. The filled-in products are then ligated to SplinkTA, a modified splinkerette with a thymidine (filled star) at the end which can exactly pair to the adenosine at the end of extension products. Subsequently, two consecutive rounds of PCR are performed followed by PCR products cloning and sequencing. (B) SplinkBlunt-PCR protocol. Genomic DNA (horizontal lines) is digested with restriction enzymes ( $\mathrm{X}, \mathrm{Y}$, and $\mathrm{Z}$, in this case) in a similar way to that described in panel A. Following denaturation of the digested double-stranded DNA, a $5^{\prime}$ biotinylated provirusspecific primer (closed circles) is annealed to its cognate region on single-stranded DNA fragments and immediately extended with $P f u$ DNA polymerase to produce blunt ends, which are subsequently ligated to SplinkBlunt. The biotin-bearing DNA fragments are then purified with streptavidin magnetic particles prior to two rounds of PCR and product cloning as performed in panel A.

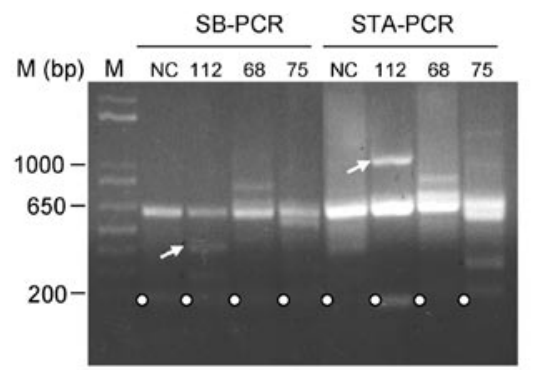

Figure 2. Comparison of PCR band patterns amplified from leukemias using SplinkBluntPCR and SplinkTA-PCR (STA-PCR) protocols. $\mathrm{B} 112, \mathrm{~B} 68$, and $\mathrm{B} 75$ are $\mathrm{BXH}-2$-derived leukemia samples. $\mathrm{NC}$, healthy $\mathrm{BXH}-2$ mice tail DNA used as a control; $\mathrm{M}, 100$-bp DNA marker. The white arrows indicate the differential bands. The white dots indicate all the PCR bands seen on the gel. The size of DNA marker in base pairs is noted to the left.

to-full recovery of PISs from BXH-2 tumors. Since the BXH-2 tumor has two germline proviral insertions, which heavily interfere with isolation of somatically acquired proviral insertions, the isolation of BXH-2 insertion sites was previously challenging and represented a major barrier to leukemogenesis study using this mouse model. Thus, our

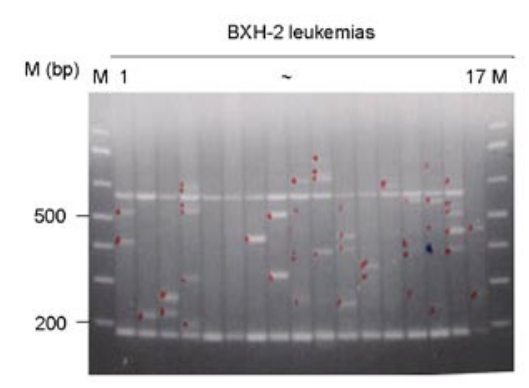

Figure 3. Application of PCR-based protocols to large-scale screen of proviral insertions in BXH-2 leukemias. Lanes 1-17 are leukemia samples. The dots denote the PCR products representing variant somatically acquired proviral insertions. M, 100-bp DNA ladder.

protocols represent an improved method for PIS-cloning in $\mathrm{BXH}-2$ tumor.

Our results have demonstrated that SplinkBlunt-PCR and STA-PCR provide an efficient, reliable, and less biased PIS-mapping method. Compared with several reported PIS cloning strategies $(2,10,12)$, STAPCR and SplinkBlunt-PCR can easily recover more PISs with $>95 \%$ specificity (data not shown) within a few days. We believe that the increased efficiency in PIS cloning can be ascribed to incorporation of several advantageous features besides the design of highly retrovirus-specific primers: (i) digestion of genomic DNA with multiple restriction enzymes in one PCR cloning experiment results in more amplifiable DNA fragments, reduces PIS-cloning bias introduced by single restriction enzyme-dependent digestion, saves time and effort by alleviating the need for more reactions and linkers, which are necessary for single enzyme-based protocols, and likely increases the specificity of PCR; (ii) adoption of splinkerettes designed as a hairpin structure on one strand rather than a central mismatch eliminates the end-repair priming phenomenon and thereby results in greater efficiency in LM-PCR $(24,25)$; (iii) formation of the SplinkTA in STAPCR with an extended thymidine at the end increases ligation efficiency, maximizes its compatibility with a variety of fragments generated by different restriction enzymes digestion, and decreases the likelihood of forming tandem blunt ligation products as well; (iv) inclusion of primer extension and purification steps in SplinkBlunt-PCR gets rid of the majority of non-template DNA, which would adversely affect efficiency of downstream PCR; and (v) relatively short experimental procedures allow completion of mapping PISs, typically within 10 days.

On the wave of increasing interest in the cloning of unknown genomic DNA flanking insertional mutagens, applications of insertional mutagenesis have demonstrated their power in discovering disease genes. To maximize information obtained from the use of this strategy, it is crucial to capture a genome-wide profile of PISs. The insertion site cloning protocols that we have described in this report provide a useful, time-saving, and efficient tool. We expected that with minor modification, these protocols can be readily adapted to identify PISs in other retrovirally induced cancer models or to map insertion sites of other types of insertional mutagens, such as transposons and retrotransposons (24-28). The techniques can also be applied 


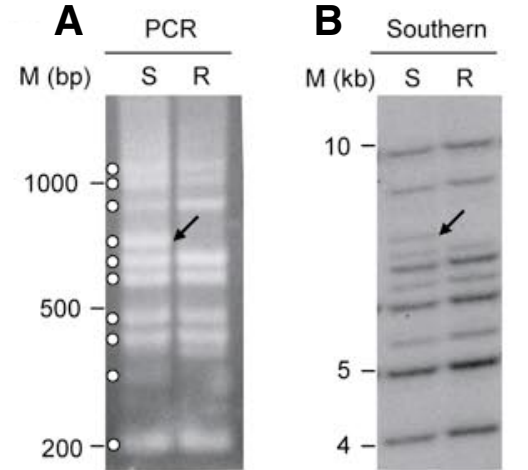

Figure 4. Comparison between SplinkBluntPCR and Southern blot analysis in detecting differential proviral insertions. The genomic DNA from Ara-C-sensitive cell line B117P (lane $\mathrm{S}$ ), used as a passage control, and Ara-C-resistant cell line B117H (lane R), were subjected to proviral insertion cloning PCR protocol (A) or Southern blot analysis (B). Arrows indicate the differential insertions. White dots indicate all the PCR bands seen on the gel.

to determine end points of genomic DNA fragment, chromosomal breakpoints involved in deletion or translocation, intron-exon junctions, and gene regulatory regions.

\section{ACKNOWLEDGMENTS}

This work is sponsored by National Institutes of Health grant no. 5R01CA081051-03 and the National Cancer Institute grant no. U01-CA652-6054. We would like to thank Dr. Kirk J. Wangensteen for his editing of this manuscript.

\section{COMPETING INTERESTS STATEMENT}

The authors declare no competing interests.

\section{REFERENCES}

1. Badarinarayana, V., P.W. Estep 3rd, J. Shendure, J. Edwards, S. Tavazoie, F. Lam, and G.M. Church. 2001. Selection analyses of insertional mutants using subgenic-resolution arrays. Nat. Biotechnol. 19:1060-1065.

2.Li, J., H. Shen, K.L. Himmel, A.J. Dupuy, D.A. Largaespada, T. Nakamura, J.D. Shaughnessy, Jr., N.A. Jenkins, et al. 1999. Leukaemia disease genes: large-scale cloning and pathway predictions. Nat. Genet. 23:348-353.
3. Mikkers, H., J. Allen, P. Knipscheer, L. Romeijn, A. Hart, E. Vink, and A. Berns. 2002. High-throughput retroviral tagging to identify components of specific signaling pathways in cancer. Nat. Genet. 32:153-159.

4.Suzuki, T., K. Minehata, K. Akagi, N.A. Jenkins, and N.G. Copeland. 2006. Tumor suppressor gene identification using retroviral insertional mutagenesis in Blm-deficient mice. EMBO J. 25:3422-3431.

5.Suzuki, T., H. Shen, K. Akagi, H.C. Morse, J.D. Malley, D.Q. Naiman, N.A. Jenkins, and N.G. Copeland. 2002. New genes involved in cancer identified by retroviral tagging. Nat. Genet. 32:166174.

6. Iwasaki, M., T. Kuwata, Y. Yamazaki, N.A. Jenkins, N.G. Copeland, M. Osato, Y. Ito, E. Kroon, et al. 2005. Identification of cooperative genes for NUP98-HOXA9 in myeloid leukemogenesis using a mouse model. Blood 105:784-793.

7.Castilla, L.H., P. Perrat, N.J. Martinez, S.F. Landrette, R. Keys, S. Oikemus, J. Flanegan, S. Heilman, et al. 2004. Identification of genes that synergize with Cbfb-MYH11 in the pathogenesis of acute myeloid leukemia. Proc. Natl. Acad. Sci. USA 101:4924-4929.

8. Lund, A.H., G. Turner, A. Trubetskoy, E. Verhoeven, E. Wientjens, D. Hulsman, R. Russell, R.A. DePinho, et al. 2002. Genome-wide retroviral insertional tagging of genes involved in cancer in Cdkn2a-deficient mice. Nat. Genet. 32:160165.

9. Largaespada, D.A. 2000. Genetic heterogeneity in acute myeloid leukemia: maximizing information flow from MuLV mutagenesis studies. Leukemia 14:1174-1184.

10. Blaydes, S.M., S.C. Kogan, B.T. Truong, D.J. Gilbert, N.A. Jenkins, N.G. Copeland, D.A. Largaespada, and C.I. Brannan. 2001. Retroviral integration at the Epi1 locus cooperates with $\mathrm{Nf} 1$ gene loss in the progression to acute myeloid leukemia. J. Virol. 75:9427-9434.

11. Triglia, T., M.G. Peterson, and D.J. Kemp. 1988. A procedure for in vitro amplification of DNA segments that lie outside the boundaries of known sequences. Nucleic Acids Res. 16:8186.

12.Hansen, G.M., D. Skapura, and M.J. Justice. 2000. Genetic profile of insertion mutations in mouse leukemias and lymphomas. Genome Res. 10:237-243.

13.Shen, H., T. Suzuki, D.J. Munroe, C. Stewart, L. Rasmussen, D.J. Gilbert, N.A. Jenkins, and N.G. Copeland. 2003. Common sites of retroviral integration in mouse hematopoietic tumors identified by high-throughput, single nucleotide polymorphism-based mapping and bacterial artificial chromosome hybridization. J. Virol 77:1584-1588

14. Largaespada, D.A., J.D. Shaughnessy, Jr., N.A. Jenkins, and N.G. Copeland. 1995. Retroviral integration at the Evi-2 locus in BXH-2 myeloid leukemia cell lines disrupts Nf1 expression without changes in steadystate Ras-GTP levels. J. Virol. 69:5095-5102.

15. Yin, B., K. Morgan, D.E. Hasz, Z. Mao, and D.A. Largaespada. 2006. Nfl gene inactivation in acute myeloid leukemia cells confers cytarabine resistance through MAPK and mTOR pathways. Leukemia 20:151-154.

16. Yin, B., S.C. Kogan, R.A. Dickins, S.W. Lowe, and D.A. Largaespada. 2006. Trp53 loss during in vitro selection contributes to acquired Ara-C resistance in acute myeloid leukemia. Exp. Hematol. 34:631641.

17.Herr, W. and W. Gilbert. 1983 Somatically acquired recombinant murine leukemia proviruses in thymic leukemias of AKR/J mice. J. Virol. 46:70-82.

18. Altschul, S.F., W. Gish, W. Miller, E.W. Myers, and D.J. Lipman. 1990 Basic local alignment search tool. J. Mol. Biol. 215:403-410.

19. Clark, J.M. 1988. Novel non-templated nucleotide addition reactions catalyzed by procaryotic and eucaryotic DNA polymerases. Nucleic Acids Res. 16:9677-9686.

20. Hu, G. 1993. DNA polymerase-catalyzed addition of nontemplated extra nucleotides to the $3^{\prime}$ end of a DNA fragment. DNA Cell Biol. 12:763-770.

21.Jenkins, N.A., N.G. Copeland, B.A. Taylor, H.G. Bedigian, and B.K. Lee. 1982. Ecotropic murine leukemia virus DNA content of normal and lymphomatous tissues of BXH-2 recombinant inbred mice. J. Virol. 42:379-388.

22. Yan Yuanxin, A.C., Li Li, Gu Jiayu, Tan Guihong, and Chen Zhangliang. 2003. T-linker-specific ligation PCR (T-linker PCR): an advanced PCR technique for chromosome walking or for isolation of tagged DNA ends. Nucleic Acids Research. 31:e68.

23.Schmidt, M., G. Hoffmann, M. Wissler, N. Lemke, A. Mussig, H. Glimm, D.A. Williams, S. Ragg, et al. 2001. Detection and direct genomic sequencing of multiple rare unknown flanking DNA in highly complex samples. Hum. Gene Ther. 12:743-749.

24. Devon, R.S., D.J. Porteous, and A.J. Brookes. 1995. Splinkerettes--improved vectorettes for greater efficiency in PCR walking. Nucleic Acids Res. 23:1644-1645.

25. Ivics, Z., P.B. Hackett, R.H. Plasterk, and Z. Izsvak. 1997. Molecular reconstruction of Sleeping Beauty, a Tc1-like transposon from fish, and its transposition in human cells. Cell 91:501-510.

26. Golling, G., A. Amsterdam, Z. Sun, M. Antonelli, E. Maldonado, W. Chen, S. Burgess, M. Haldi, et al. 2002. Insertional 


\section{Short Technical Reports}

mutagenesis in zebrafish rapidly identifies genes essential for early vertebrate development. Nat. Genet. 31:135-140.

27. Collier, L.S., C.M. Carlson, S. Ravimohan, A.J. Dupuy, and D.A. Largaespada. 2005. Cancer gene discovery in solid tumours using transposon-based somatic mutagenesis in the mouse. Nature 436:272-276.

28. Dupuy, A.J., K. Akagi, D.A. Largaespada, N.G. Copeland, and N.A. Jenkins. 2005. Mammalian mutagenesis using a highly mobile somatic Sleeping Beauty transposon system. Nature 436:221226.

Received 3 January 2007; accepted 28 March 2007.

Address correspondence to Bin Yin, 6-160 Jackson Hall, 321 Church St. S.E., Minneapolis, MN 55455, USA. e-mail: binxx002@umn.edu

To purchase reprints of this article, contact: Reprints@BioTechniques.com 